\title{
Universal Classifications, National Approaches and Specific Situations: A Comparative Study on the Conceptualization of Nodding Syndrome in Uganda and Tanzania
}

\author{
Karin van Bemmel ${ }^{\mathrm{a} 1}$ and Kim van der Weegen ${ }^{\mathrm{b}}$ \\ ${ }^{\mathrm{a}}$ Department of African Languages and Cultures, Ghent University, Belgium; ${ }^{\mathrm{b}}$ Department of Cultural \\ Anthropology, Utrecht University, The Netherlands
}

\begin{abstract}
This paper presents a comparative study on conceptualizations of the poorly understood nodding syndrome (NS) in Uganda and Tanzania. NS has been constructed as a biomedical category to serve global health discourse as well as national contexts of managing the condition. We look into the shifting meanings and conceptualizations of NS in the affected areas of Kitgum (UG) and Mahenge (TZ) district. The perceived universality of biomedical classifications is problematized as conflicting with the specific contexts of lucluc and kifafa cha kusinzia. Reconciliation proves to be challenging, poignantly evoking the cultural construction as such of any medical condition.
\end{abstract}

Keywords: nodding syndrome, conceptualization, medical anthropology, Uganda, Tanzania

\section{Introduction}

Although briefly described in the 1960s and occurring in three relatively small areas in Uganda, Tanzania and South Sudan, the poorly understood nodding syndrome (NS) has received increasing international attention over the past decade. Medically defined as a seizure disorder, the syndrome manifests itself in episodes of head nodding, combined with stunted growth and cognitive decline in children between five and fifteen years old (Dowell et al. 2013). So far, no definitive cause has been identified and little is known about its course and cure. The nodding episodes are mainly triggered by food and cold weather and in many cases the patient eventually develops more complex partial and generalized seizures (Winkler et al. 2008, 2014). NS itself does not seem to be lethal, but patients have died due to accidents during seizures that led to drowning or burning (Korevaar and Visser 2012, 2). In addition, several cases of death as a result of status epilepticus have been reported during fieldwork in northern Uganda. It is estimated that 1.687 (Iyengar et al. 2014) up to 3.094 (DGHS 2012) children in Uganda are affected by NS and a total of 5.000-10.000 people in East Africa (Idro et al. 2013). As these numbers indicate, there is not much knowledge on the actual prevalence

\footnotetext{
${ }^{1}$ Corresponding author. Email: Karin.VanBemmel@UGent.be
} 
of the syndrome and specific information on the amount of cases in Tanzania and South Sudan is not available.

Several biomedical hypotheses on the etiology of NS circulate in the academic arena. The possibility of a relationship between nodding syndrome and onchocerca volvulus is frequently mentioned, because many of the NS-areas are highly infested with OV (Winkler et al. 2008, 2008). It has also been suggested that NS is a genetic syndrome, given the positive family history with epilepsy found in Tanzania (Winkler et al. 2014, 92). Other potential causal factors include mycotoxins (Spencer et al. 2013), post-traumatic stress (Musisi et al. 2013) and a neurotropic virus (Colebunders et al. 2014). The course of NS on the long term is unknown, as well as prognosis and optimal treatment. Currently, patients are treated with antiepileptic drugs (AEDs) to control the seizure activity. Several types of AEDs are used in the different countries and there is no clarity about which medication is most effective (Dowell et al. 2013, 1383). In addition, there is a lack of guidelines, tools, and medical technologies, making it difficult to confirm a diagnosis of NS and to distinguish it from other medical conditions (Van Bemmel, Derluyn, and Stroeken 2014).

The academic literature predominantly presents nodding syndrome from a biomedical point of view and leaves little room for the social context in which it occurs. According to Lewis-Fernández (1996) there is limited usefulness of purely descriptive diagnostics and a fallacy of universalistic course predictions and outcome measures. He pleads for an intensification of research on the epidemiology of indigenously defined syndromes as a powerful way of problematizing the universality of established Western nosologies (1996, 138). In the current study, a social constructionist approach to illness is applied, whereby the specific contextual factors of NS in Uganda and Tanzania are explored and attention is paid to the negotiation of categories. Determining which biological states are labelled as disease is a complex process of social construction (Conrad and Barker 2010, 67), and we should be aware of the unwarranted assumption that illness-categories have the same meaning in every cultural context.

Previously, focus has been put on the conceptualization of nodding syndrome in Uganda (Van Bemmel, Derluyn, and Stroeken 2014; Van Bemmel 2016a, 2016b). The comparison in the current paper enables the exploration of different (inter-)national and local discourses that reflect the diversity of negotiations. It offers insight into the construction of NS as a biomedical category and the meaning of head nodding symptoms in national and local contexts. Diverse layers of meaning are emphasized, illustrating how the presumed universality of a biomedical concept of NS does not hold when situated in different affected settings. It is the encounter between actors, discourses, and practices that leads to the unique and continuously changing meaning of nodding syndrome in Uganda and Tanzania.

\section{Methods}

Ethnographic fieldwork is characterised by the researchers' prolonged residence among the research population, participation and observations in the field in order to get a holistic view of the subject that is being studied (Sluka and Robben 2012, 7). When studying the construction of nodding syndrome this approach to fieldwork becomes slightly problematic. First, we must be careful not to attribute conclusive explanatory power to a homogeneous idea of NS as universal phenomenon. At the same time, we should not overlook the discursive 
power of this universalizing tendency. Secondly, the actors determining the field of NS cannot be limited to one (or two) geographically circumscribed area(s), instead it is the interaction between many scattered actors and sites that should be explored. Marcus (1995, 105) describes multi-sited fieldwork as being designed around 'chains, paths, threads, conjunctions, or juxtapositions of locations in which the ethnographer establishes some form of literal, physical presence'. At the centre of these paths is the object of study: the medically labelled nodding syndrome, but also the concepts of lucluc in northern Uganda and kifafa cha kusinzia in central Tanzania. In this paper they are approached as a collection of associations and explanations that connect a variety of actors. It is the first time that a qualitative comparison is made between NS affected areas and we refer to this research as multi-sited in the sense that it is composed of multiple sites organised around multidirectional connections ${ }^{2}$. Thereby we shift between international, national and local levels and embrace the indeterminate and fragmentary character of NS-narratives that circulate in the different areas.

The idea of multi-sitedness does not only apply to geographical scales, but the narratives on NS circulate through qualitatively different sites, such as the compound, the family, healer's practices, media and so on. Since nodding syndrome is constructed at different levels, it is helpful to make a conceptual distinction between disease and illness. Disease can be understood as biological abnormalities in the structure or functions of the body, reinterpreted as objective symptoms by medical practitioners (Hanna \& Kleinman 2013, 20). This is for example done in the construction of NS as a biomedical category to serve global health discourse. Illness refers to the subjective experience of certain socially disvalued states and the social meaning attributed to them (Conrad \& Barker 2010, 67). It can therefore stretch beyond what is considered to be a disease according to the biomedical model.

The study of the construction of NS in different sites, asks for a variety of research methods. Apart from the study of written reports, news articles and academic literature concerning NS, the descriptions in this paper are based on fifteen months of data gathering in northern Uganda (2012, 2013 and 2014, KvB) and three months of explorative fieldwork in Tanzania (2014, KvdW). Although the head nodding symptoms were approached with a neutral stance, we should acknowledge the researchers as actors in the meaning making process. Through the exchange of knowledge during (both verbal and non-verbal) encounters of ethnographic fieldwork, the researchers inevitably took part in the production of meaning. Their presence, the questions they asked and their interest in the syndrome all contributed to its conceptualization. However, through the combining of research strategies and the inclusion of different narratives, the outcomes of this study are less dependent on a singular perspective.

The main techniques for data collection were participant observations and informaland semi-structured interviewing. Part of the fieldwork is done in the cities of Kampala (UG) and Dar es Salaam (TZ), more specifically in the offices of governmental representatives, global health actors and during an international conference on nodding syndrome. In both cities semi-structured interviews were conducted in English with actors from World Health

\footnotetext{
${ }^{2}$ In the future it would be interesting to compare data across all three affected countries. Unfortunately, no anthropological studies on nodding syndrome in South Sudan are currently known to us.
} 
Organization (WHO), Centers for Disease Control and Prevention (CDC), several nongovernmental organizations (NGO's), the Ministry of Health and academic researchers involved in the investigation of NS. Interviewees were asked about their attitudes and perceptions towards the head nodding symptoms and attention was paid to processes of labelling. Even though no NS-cases have been reported from Kampala and Dar es Salaam, the actors in these cities have powerful voices in the construction of NS.

Another set of data is gathered in the affected communities in northern Uganda and south-central Tanzania (Figure 1), where actors dealing directly with NS were interviewed and observations were made. In Uganda, fieldwork took place in Kitgum district, an area highly affected by nodding syndrome. Fifty in-depth interviews were conducted with affected families at their homes and in the hospital. Furthermore, forty interviews took place with health workers, traditional healers, local area leaders, media representatives and community members. Participatory observations were made at NS treatment centres, during outreaches, healing ceremonies and at the homes of affected children. In Tanzania, fieldwork took place in the Vigoi division in Ulanga district. The Mahenge Epilepsy Clinic is a central point in the management of NS and observations took place during consultations between health workers and patients. Semi-structured, informal and focus group interviews were conducted at the homes of twelve NS affected families and with twenty people suffering from epilepsy. In addition, traditional healers, several community members, health workers, catholic priests and village leaders were interviewed. All interviews conducted in Uganda and Tanzania took place after receiving verbal informed consent and with help of a translator in Leb Acholi (UG) and Kipogoro and Kiswahili (TZ). Each interview took between 30 and 70 minutes and was afterwards anonymised and translated into English for coding. Thematic analysis of the data took place through the identification of key concepts in the interviews. In this article findings on the conceptualization of nodding syndrome are presented.

The context in which NS exists largely influences its construction. Moving away from solely biomedical explanations, this paper shifts from the medical category of nodding syndrome to concepts circulating in the affected areas. Hereby we illustrate how NS is constructed through encounters between different actors, discourses, and practices. First, focus is put on the construction of nodding syndrome as a biomedical category to serve global health discourse. Furthermore, attention is paid to the national contexts in which the syndrome exists and the significance of NS as category for policymakers and practitioners. Thereafter, focus is put on the shifting meanings and conceptualizations of NS in the two affected areas of Kitgum (UG) and Mahenge (TZ) district. Although global health actors have powerful voices directing the definition that is used, reconciliation with the specific context of the syndrome proofs to be challenging.

\section{Nodding syndrome as a biomedical category}

\section{First accounts}

The involuntary and repetitive dropping of the head characteristic of NS was first reported by Dr. Jilek-Aall in Tanzania in the 1960s. During her medical work in Mahenge district, the Norwegian physician noticed a high prevalence of epilepsy among the Wapogoro, an ethnic group living in south-central Tanzania. The majority of the 175 patients in her study suffered from fairly typical grand mal seizures. A small part (27) of her research population however 
presented with fits which could not be identified exactly as either akinetic attacks or myoclonic petit mal. Jilek and Jilek-Aall report that in these cases 'the child suddenly drops his head to the chest, then raises it up again' $(1970,46)$. This type of seizure was only found in children and frequently occurred in the morning shortly after awakening. According to their report, the descriptive term used by local people was amesinzia kichwa, Kiswahili for 'nodding of the head' (Jilek and Jilek-Aall 1970, 46).

In 2001, the WHO was invited to investigate 'an epidemic of an illness characterized by episodes of repetitive nodding of the head' in the former southern Sudan (Tumwine et al. 2012, 242). The local Moru people had noticed the syndrome in 1991 and referred to it by the name adravu legnaro; 'a disease of eating ugali', which points to the association of nodding episodes with the act of eating local food (Spencer et al. 2013, 191). In northern Uganda, head nodding was noticed by the local populace around 1998 and referred to as lucluc (nodding) or yengo wic (shaking head). The symptoms came up during a period of war between the Ugandan government and Lord's Resistance Army (LRA). The first biomedical investigations took place in 2009, led by the Ministry of Health in cooperation with the WHO and CDC.

\section{Global health}

Although local accounts of head nodding existed in the affected area, Jilek-Aall's writings are the first known reports in which it is described as a biomedical condition. It was only after the discovery of similar symptoms in South Sudan and Uganda, that the nodding episodes gained international interest and studies started focusing on NS as a distinct medical condition. At this time health organisations, scientists, and politicians active in the arena of global health became involved in the construction of nodding syndrome on an international level.

Global health as a policy took shape when large institutions started coordinating international health initiatives. In the early stages of the global health agenda, nation states were the main source of authority in terms of setting priorities and allocating resources, but this changed after the United Nations' Millennium Development Goals made health one of its focus points for development (Biehl and Petryna 2013, 6). From then on, a variety of actors with different motives and backgrounds jumped on board under the banner of humanitarianism, development and security. Global health therefore is not only the terrain of medical disciplines involved in the practice of health care, but also the terrain of politics, power, knowledge, social organization and economics (Langwick, Dilger, and Kane 2012, 1). Prototypes of disorders predominantly derive from those with the power to reach large audiences and to be seen in research. National and private medical institutions, NGO's, pharmaceutical companies and national governments are now all possessors of what is called biopower, defined by Fassin as a 'normalizing power over life' $(2011,185)$. Knowledge about the body gives them the (bio)power to decide on the distribution and access to healthcare, medicine, and financial aid (Hanna and Kleinman 2013, 26). Although biomedical diagnoses have major influences on people's lives, the individual does not have a say in the actual labelling: it is the medical professional possessing the knowledge and power to decide.

It is not only the ability to define disease that made global health actors powerful in the construction of nodding syndrome, it is also the construction of biomedical knowledge as being universal, evident in the utopian vision of 'health for all'. During the formation of a medical definition of NS, science was used as a universal language aspiring to 'articulate all 
things in a common language' and 'to be knowledge for everyone, regardless of where and how we live, a universal human legacy' (Fuller 2015, 61). Through these universal claims, medical discourse is able to influence how people behave towards their bodies in illness and in health (Conrad and Barker 2010, 69). It is therefore important to consider the processes through which biomedical knowledge is produced.

\section{The creation of a case definition}

Over hundred actors were engaged in a powerful NS-discourse during the International Scientific Meeting on Nodding Syndrome held in Kampala in mid-2012. The meeting was organised by the WHO in collaboration with CDC, UKAID and the Ugandan Ministry of Health (MoH). Scientists, politicians, and NGO's involved in the medical field were invited and plans were made for further investigations and collaborative actions in regard to NS. Since different labels were used to refer to what seemed to be a similar set of symptoms, it was decided that from then on 'nodding syndrome' would be the most appropriate name. The participants also came up with a case definition that was to be used in all affected countries to 'ensure consistency in identification of cases and comparable research results' (WHO 2012, 20). This was done by elaborate discussions on the inclusive criteria of a nodding syndrome case. During the sessions, a word-document was projected on a large screen, enabling all attendees to follow (and take part in shaping) the definition-making process. Discussions for example focussed on age restrictions, the frequency of nodding per minute and the desirability of including pre-nodding abnormalities. Secondly, much time was spent on the actual creation of the case definition, whereby linguistic issues came to the fore. The division into 'major' and 'minor' criteria was debated, as well as the difference between 'observed' and 'reported' episodes of nodding and the (in)advisability of including footnotes. On the projected worddocument all attendees could follow the adaptations, until consensus was reached over the description of a nodding syndrome case.

The international scientific meeting and the actors involved exemplify how the construction of NS takes place on a global platform; together the actors had the power to define NS and choose the label to be used on an international scale. Nodding syndrome became categorized and differentiated from other afflictions: it became 'diagnosable' and was classified as a neglected tropical disease by the WHO. This gave rise to novel connections between families, hospitals and health workers in the affected areas and researchers, funding organizations and institutes (e.g. WHO and CDC) at the other side of the world. Spaces that were not necessarily connected to each other, were now transformed into overlapping, transnational zones of action. It is this construction that caused a shift in meaning of what was considered to be a type of epilepsy in Tanzania, an eating-related syndrome in South Sudan and a set of unexplained symptoms in Uganda, to a distinct medical condition called nodding syndrome. Currently, the term 'nodding syndrome' and its biomedical case description are reflected in academic literature, research reports and on websites from global health institutions. But when looking at the intersections between the universalising logic of biomedicine, with its focus on international classifications, and the particular settings of NS in Uganda and Tanzania, defining nodding syndrome becomes more complicated.

\section{Nodding syndrome in Uganda and Tanzania}




\section{National policies}

During the scientific meeting, Ugandan and Tanzanian actors sat down together for the first time to discuss the prevalence of the poorly understood syndrome in both areas. On the first day of the international conference, both the Ugandan Ministry of Health $(\mathrm{MoH})$ and Tanzanian Ministry of Health and Social Welfare (MoHSW) are asked to present the available data from their respective countries $^{3}$. The Tanzanian representative kicks off and within minutes it is clear that data is lacking. One of the first PowerPoint-sheets reads: 'Epidemiology of nodding syndrome in Tanzania is not documented by MOHSW'. Furthermore: 'A lack of the health system has been noticed to recognize the head nodding syndrome' leading to 'unavailability of data'. The presentation ends with a slide on gaps: 'Magnitude of the NS not known within the country (...) No case definition of NS (...) No linkage of the on-going efforts of NS studies under individual scientists and MOHSW policy guidelines and plans'. A representative from the Ministry of Health in Uganda takes over and briefs the participants on the status of NS in his country. In this presentation, the national NS coordinator reads out the Ugandan surveillance definitions and mentions that 3094 cases are found in the northern districts. He talks about several investigations conducted by a combined team of $\mathrm{MoH}$ representatives and partners. Furthermore, he mentions that criteria for casemanagement are developed, a training manual is formed $(\mathrm{MoH} 2012 \mathrm{a})$ and NS treatment centres have been set up in several districts in northern Uganda.

\section{Socio-political context}

Given the fact that head nodding was first observed in Mahenge district, it is remarkable that information from Tanzania is currently lacking. No official reports or action plans are available and the prevalence of NS in this country is unknown. A contrasting situation exists in Uganda, where an official governmental response plan and emergency budget have been released (MoH 2012b). Up to now, all new cases need to be reported to the office of the Ugandan Ministry of Health, where statistics on NS are being analysed. Furthermore, the majority of academic literature on NS is based on biomedical research that took place in Uganda. And while nodding syndrome appeared in over 250 newspaper reports in Uganda (van Bemmel 2016a), only two newspaper articles were found in Tanzania and both focus on the presence of the syndrome in Uganda (Bahati 2012; Mugerwa and Ayebazibwe 2012). During fieldwork in Dar es Salaam, most people were not aware of the existence of NS as defined by the attendees of the conference. In Kampala however, it was difficult to find anyone without at least minor knowledge -and often a highly politicized opinion- about nodding syndrome.

In order to understand the different approaches and amount of attention given to NS, it is important to keep the socio-political context in mind. The appearance of NS in Uganda took place at a time when media houses had a much larger audience than in the 1960s in Tanzania. On an international level, scientists became interested in the syndrome when its existence was reported from several places. Its importance shifted from unexplained local symptoms to a 'global health issue' and it became more attractive for international actors (and

\footnotetext{
${ }^{3}$ Representatives from the $\mathrm{MoH}$ in South Sudan were also present, but for the purpose of the comparison in this paper we focus on Uganda and Tanzania.
} 
funding agencies) to get involved. In addition, the first observations in northern Uganda were made during wartime, when heavy fighting between the LRA and Ugandan government took place. The syndrome quickly obtained political importance and the government was blamed for not paying enough attention. In 2012 a court case against the Attorney General was filed on behalf of the NS patients and heated debates took place in parliament and media, touching upon questions of responsibility and healthcare. In this way, NS became an instrument in a political struggle and was used to highlight perceived oppressions from the government Van Bemmel, Derluyn, and Stroeken 2014). It was in this context that resources for nodding syndrome were made available in Uganda. The need for public alarm in Tanzania did not arise in a similar way. The nodding symptoms were not seen as something new and no unique developments were noted to have taken place since the first observations. Furthermore, the syndrome does not have the political connotations that exist in Uganda. It was only after the discovery of cases in Uganda and South Sudan that Tanzanian health actors became actively involved.

\section{Contrasting situations}

Despite the fact that there is binding power in the international biomedical categorization, we have seen that national approaches towards NS differ. In addition, it is not clear whether NS represents the exact same phenomenon in the affected countries. The head nodding episodes are observed in all areas but other symptoms seem to differ. Lock and Nguyen challenge the assumption that human bodies are 'readily standardizable', since humans are inextricably entangled with historical, environmental, social, cultural, and political contexts $(2010,12)$. According to Mitchell and colleagues, nodding syndrome is not rapidly progressive and does not result in severe encephalopathy in most cases in Tanzania $(2013,22)$. In addition, the prevalence of NS in this country seems to be consistent over time as opposed to South Sudan and Uganda. Consequently, Winkler and colleagues $(2014,90)$ suggest that NS in Tanzania might be endemic in character, and more epidemic in character in the other two areas. During interviews in Dar es Salaam, respondents also mentioned that the symptoms and affected population in Tanzania seem somewhat different from the descriptions in Uganda and South Sudan. The actual symptoms of head nodding and generalized seizures were known for decades, but the quick cognitive decline leading to mental retardation seems to be absent in many of the Tanzanian cases. Children wandering off in a confused state of mind, as frequently described in Uganda, were not observed in Tanzania. In addition, the area was already known for a high prevalence of epilepsy and the number of affected people does not seem to be increasing, according to respondents. In fact, they often mentioned that 'nothing new' is happening in Tanzania.

The doubts about what NS in Tanzania entails reflect the friction between the internationally created definition and the specifics of NS in the national context. Although international actors have labelled the set of symptoms 'nodding syndrome' in all countries, on national level there is no consensus when it comes to the actual definition of this 'universal' medical category. As the next section will show, differences also exist between the conceptualization of NS in global health discourse and the specific areas in which the syndrome occurs. Although western concepts of illness are frequently included in local settings, they are often seen as just one of many possible lines of thinking that can be weighed 
against each other. By exploring the specific situations of nodding syndrome in Kitgum district (UG) and Ulanga district (TZ), the syndrome is presented as a dynamic, complex social construct that defies easy definition or measurement.

\section{Head nodding in Kitgum district, Uganda The clinical setting}

Kitgum district -bordered in the north by South Sudan- suffered many deaths and social disruption during the twenty years of war between the LRA and Ugandan government. Nearly ninety percent of the Acholi population in this area spent years in internally displaced persons (IDP) camps during the course of the conflict (WHO 2005). Since relative peace has returned in 2006, the majority of people have found their way back to the original homesteads. However, they are greatly affected by the atrocities that took place in this area and the scars of war are present everywhere.

One of the locations where the traumas of conflict are dealt with is the mental health clinic at the district government hospital. This is also the department where nodding syndrome patients are consulted and collect their medication on a monthly basis. In 2012, Kitgum hospital was assigned the task of NS case management. With support from the Ministry of Health a treatment center was set up within the hospital ground, consisting of an outpatient screening site and an inpatient department for admission of severe cases. Although a large signpost offering 'nodding disease services' still points towards the solid building of the current children ward, it was only for a short period of time that the NS center was located here. Within a few months after its joyful opening, patients were shifted to a ward at the back of the hospital area, close to the mortuary. Seated in front of the building, one could frequently observe how corpses were carried in and out of the small construction. Two monitor lizards occasionally popped up, wandering around in the surrounding grass. Next to the ward, caretakers were busy preparing posho meals and siblings of the admitted patients ran between the small cooking fires, all patiently waiting for the day they could return home. In mid-2014, the patients shifted location for the third time. Currently, a former storage room of a humanitarian organization functions as the NS treatment center. Like the mental health clinic, its location is separated from the rest of the hospital and a tall fence surrounds both buildings.

The opening of the treatment center was part of a three-year during NS-response plan launched by the $\mathrm{MoH}$ in 2012. The central monitoring of the plan is done by the Ministry of Health and a national coordinator was appointed. In addition, a national NS taskforce has been formed, mainly consisting of doctors and psychiatrists who create guidelines and undertake research and supervision. The implementation of activities in the affected districts is led by the district health offices. District NS taskforces have been set up and are supposed to share ideas with regional stakeholders on a regular basis, but meetings seldom take place. Furthermore, a selection of health workers received a special training on how to handle NS cases. Throughout early interviews in 2012, nurses mentioned feeling fearful and hesitated to come close to the affected children. Nowadays fear has reduced, because scientists are convinced that the syndrome is not contagious and new cases of NS are rarely seen. People in the affected villages occasionally report new cases, which is contradicted by representatives of the $\mathrm{MoH}$. 
A diagnosis of nodding syndrome is indicated as ' $\mathrm{NS}$ ' in the hospital record book, as well as in the personal booklets that people take home. When the patient also suffers from epileptic seizures the label 'NS+' or 'NSP' (Nodding Syndrome Plus) is given, and a diagnosis of epilepsy is marked as ' $\mathrm{E}$ '. A difference is made in prescribed medication for patients with a diagnosis of epilepsy and NS/NSP; the nodding patients preferably receive Sodium Valproate and people with epilepsy are put on Carbamazepine and sometimes take Phenytoin or Phenobarbital ${ }^{4}$. Medication is made available through supply from the $\mathrm{MoH}$ and the management of NS(P) and epilepsy is interlinked. On a regular basis the hospital statistics are send to the $\mathrm{MoH}$ office in Kampala, where data on nodding syndrome are analyzed. According to the hospital book, approximately two hundred patients (NS, NSP and E) have been admitted at the nodding syndrome ward in 2012. The number of admissions decreased in 2013 when around seventy children were admitted, many of them suffering from severe burns.

Kitgum hospital provides as a referral NS treatment centre for severe cases from Lamwo and Pader district. Complicated cases can also be sent to the regional hospital in Gulu and to Mulago hospital in Kampala for further management. In addition to case management at the hospital, the nurses and clinical officer are supposed to visit three outreach sites in Kitgum district on a weekly basis. The MoH supplied the NS-team with a van and motorcycles, but the outreaches are irregularly conducted due to a frequent lack of fuel, medication, accessible roads and available staff.

\section{Illness experiences}

The households affected by nodding syndrome are mainly situated along riversides. Here, the syndrome is known as lucluc, which refers to the episodes of head nodding ${ }^{5}$. Other mentioned symptoms are saliva drooling and a state of confusion. Caretakers frequently report that the affected children see people -invisible to others- approaching them and hear voices calling their names. The images are often related to war, e.g. soldiers that are carrying rifles or rebels with pangas.

Lucluc is differentiated from lirlir (two lirlir/lili), which is associated with uncontrollable jerking movement of the arms and legs, falling, frothing, temporary confusion and incontinence. Lirlir (translated as epilepsy by local health workers) has been present in the area for a long time but lucluc is a new illness that came up during the period of war, according to the local population.

A variety of explanatory models circulate about the etiology of lucluc. The majority of respondents mention the interference of cen, revengeful spirits of people who died in a violent way. Because numerous people were murdered during the war, many cen are now considered to roam the area. Furthermore, the use of weapons and bombs during the recent insurgency is suggested to play a causal role. The small, black flies (transmitting onchocerca volvulus) residing near rivers are also considered as possible etiological factor, following suggestions from visiting researchers. However, the hypotheses posed by global health actors are frequently rejected in the local context, where conflict or spirits are central in most

\footnotetext{
${ }^{4}$ In addition, the patients receive Folic Acid and Multivitamins. When the person does not respond well to the medication, the medical doctors are supposed to offer alternative treatment.

${ }^{5}$ Other names that are sporadically used are two luj (nodding sickness) and yengo wic (shaking head).
} 
explanations. The grandmother of two affected children for example states; 'People say that it's caused by black flies, but it's not true. Others say it's the food we ate in the camps. But I don't believe that is the cause.' (Van Bemmel and Aalten 2016, 12:00). In addition, the mother of a severely sick girl mentions: 'Some say it's black flies [that caused NS], but they were always here. It's the war that brought it' (2016, 19:45). These comments illustrate that ideas are not formed in isolation, but are shaped in dialogue with others. Frustration prevails towards biomedical scientists that spend a short amount of time in the affected area and fail to explain and share their research findings. According to respondents, this leads to the inability to understand what lucluc entails. In addition, distrust of governmental actors occasionally leads to the reluctance to accept help.

Most of the affected people stay at home, where the social effects of the syndrome can be devastating. A number of children are occasionally tied to trees to prevent them from wandering away in a confused state of mind, several afflicted children have been sexually harassed and some of the victims were impregnated. In addition, the past conflict has a major impact on the family situations in the area. Fathers are often not around and there are many reports on substance abuse. Caretakers report feelings of sadness, isolation, fear and hopelessness. Furthermore, the illness interferes with their income generating activities, because the children have to be under continuous observation. The situation is aggravated by the fact that NS frequently affects several children within one family. In some areas the caretakers have set up small saving cashbox groups. Besides building a financial support network, they often discuss the status of the syndrome during their monthly gatherings. In one of the affected villages, a group of caretakers has formed a choir and asks for attention to lucluc. The songs for example address the issue of children falling into fire or water during attacks (Okony Tumangu Tim 2014a), dealing with the death and burials of their loved ones (2014b) and the mysterious cause of the affliction (2014c). Because most of the affected families live close to each other, they communicate on a daily basis and advise each other in regard to the complications that the illness brings.

In addition to support from family members and health centres, the affected children seek help from traditional healers. Some children visit an ajwaka, a spiritual medium who facilitates a dialogue between the afflicted person and the revengeful spirit(s). In order to treat the affected person, the spirit needs to be appeased and send out of the body. Several families collected money for a joint traditional healing ceremony in Kitgum district, but no improvement was reported afterwards. Islamic and Christian healers have also been treating affected children, by using anointed water and prayers to chase out jinn and devils, and different types of herbal medication have been distributed. However, since definite answers on cause and cure remain lacking, the quest for treatment continues (Van Bemmel 2016b).

Deborah (pseudonym) and her daughters live in one of the most affected villages in northern Uganda and have been looking for treatment over the past decade. Their story provides an illustration of the experience of lucluc in Kitgum district:

After an approximate forty-minute ride on a motorbike, we reach the household of Deborah. Her husband has recently died and she takes care of seven children by herself. When they were younger they did not experience any health problems, but now two of her daughters are suffering from lucluc. Akello is 15 years old and started having 
episodes of head nodding in 2005 during her stay in an IDP camp. Later, she also started falling down (reto ping) and shaking (dancing) the body (miel kom). Her sister Idah (now 11 years olds) reported the first symptoms in 2009, after the family had returned home from the camp. She frequently has an episode of lucluc, but unlike Akello does not suffer from falling down. In both girls, the nodding occurs on a daily basis in response to food or in the early morning when it is cold, but the episodes also come without trigger. Deborah is afraid that her daughters will get hurt and doesn't allow them to come near the fireplace or go to the river. The sisters attended a healing ceremony in which a sheep was killed and cen was being addressed, but their condition did not improve. Currently, they receive Sodium Valproate from the hospital outreach team, but mother reports that 'the medication keeps on changing' and that there is no improvement. She suggests that lucluc comes as a result of fighting in the war and also mentions that it is a spirit that brings the sickness. At the end of our visit, mother emphasizes the importance of 'finding medication that can help them'.

The case of Deborah's family illustrates how stories on conflict, spiritual actors and biomedicine are incorporated into the experience of lucluc. Multiple explanatory models and help seeking attempts based on different discourses are mentioned, pointing to the constantly shifting meaning of lucluc in Kitgum district.

\section{Head nodding in Ulanga district, Tanzania The clinical setting}

The Kilombero river, the natural border separating Ulanga district from the rest of the country, is a 12-hour bus drive away from Dar es Salaam. After crossing the river by ferry, the journey continues for hours on a dirt road, up to the mountains of Mahenge. Mahenge is the main town of Ulanga district, situated in the Morogoro region in south-central Tanzania. Like Kitgum, it has all the amenities you would expect in a small town, but still it is hard not to feel cut off from the rest of the world. A few weeks into the fieldwork a heavy rainy season hit Tanzania. The Kilombero river flooded and the ferry was out of use, meaning that no vehicles could get in or out of Ulanga. In addition, the roads connecting villages to each other and the outside world were practically impassable. Life can be tough for the population of Ulanga, the majority of which belong to the Wapogoro ethnic group. Most people live in red mud brick houses and are forced to live off their small plots of land, because there are barely opportunities for employment. The crops people grow are mostly for their own consumption and during the dry season this often means great food shortages.

The Mahenge Epilepsy Clinic is located on the property of the government hospital in Mahenge and was founded by Jilek-Aall in 1960. Epilepsy in Tanzania is classified as a mental health condition and the epilepsy outpatient clinic serves as a district mental health clinic. Because NS is locally classified as epilepsy, it is treated here. The clinic consists of a small room situated at the back of the government hospital property. It employs two nurses who are in charge of diagnosing, treating, monitoring and evaluating patients with psychiatric conditions and epilepsy. Most patients visiting the clinic are from the Vigoi division, which covers a big mountainous part of the district so people often walk for hours to find treatment. They come to the clinic once a month to collect medication and report on their progress, the consultation usually only lasts a few minutes. 
Working together with the epilepsy clinic, Winkler and colleagues confirmed sixtytwo cases in their research on nodding syndrome in 2005 (2008, 2010). Searching through the hospital registers from recent years, twenty registered NS cases were found during the current fieldwork. In contrast to the situation in Kitgum district, patients with episodes of head nodding usually receive the code ' $E$ ', referring to epilepsy. Only two register books were found in which '+NS' (nodding syndrome) was added to the existing 'E' description. It is striking that these books were created around the time when research into NS was conducted at the clinic, while in the current registers no differentiation between epilepsy and nodding syndrome was found. It seems that the implementation of the constructed biomedical case definition does not take place as the attendees of the international conference had in mind. Besides the registers, small notebooks also enumerate diagnoses that serve as patients' files. If a patient with head nodding episodes develops generalized seizures, the diagnosis of NS is quickly changed into epilepsy. Furthermore, patients regularly stop their treatment at the clinic. All these factors make it difficult to determine how many NS-patients the clinic actually treats.

Locally not much is known about the international health discourses that take place regarding the syndrome. Occasionally the nurses at the clinic and local communities come in contact with global health actors in the form of researchers studying NS, but these visits are more sporadic than in Kitgum. During one of these meetings, nurses and patients heard about the possible link between NS and onchocerciasis. However, besides a document given to them by Dr. Jilek-Aall, no documentation about NS exists at the clinic and the interviewed nurses find it difficult to come up with a definition of nodding syndrome on request. Because little up-to-date information on NS is available to them, they rely on practical experience for a large part.

Treatment for epilepsy -and therefore NS- is distributed free of charge at the governmental clinic. The Tanzanian government provides three types of anti-epileptic drugs: Phenytoin (PHT), Phenobarbital (PB), and Carbamazepine (CBZ). Local nurses state that Carbamazepine is the medicine of choice to treat NS. However, both CBZ and PHT are regularly out of stock so patients frequently shift between different types of drugs depending on availability. A study conducted in 2009 indicates that the majority of patients are treated with the most consistently available medicine of Phenobarbital (Winkler et al. 2014, 90). During an interview, one of the nurses states that Sodium Valproate would be a better option, because it is thought to cause fewer side effects. However, in contrast to Uganda, this type of medication is not provided by the Tanzanian government.

The labelling of illness depends on the interaction between different actors. One of the nurses mentions that when foreign researchers came to the area, a separate Kiswahili term had to be developed to refer to NS in contact with the patients. The term amesinzia kichwa, as previously mentioned by Jilek and Jilek-Aall $(1970,46)$, was used by the local population to describe the actual symptom of head nodding, but not to refer to 'nodding syndrome' as a distinct medical condition. Accordingly, the term kifafa cha kusinzia was created. Kifafa is the Kiswahili word commonly used for epilepsy ${ }^{6}$ and kusinzia (to doze off) refers to the nodding

\footnotetext{
${ }^{6}$ The specific name of epilepsy with generalized seizures is kifafa kuanguka, meaning epilepsy and falling down, but usually the term kifafa is used. Kifafa cha kusinzia is considered to be the harbinger of kifafa kuanguka.
} 
of the head, as if someone is falling asleep. The transition from kifafa to kifafa cha kusinzia shows the shift in meaning of NS in Tanzania, a transition that is taking place in interaction with actors from outside. Although still seen as a form of epilepsy, nodding syndrome now has its own name in Kiswahili.

\section{Illness experiences}

Kifafa is an integral part of the construction of NS in Ulanga district, as the name kifafa cha kusinzia shows. Kifafa is associated with generalized seizures characterized by a person falling on the ground in an unconscious state combined with jerking, frothing, and incontinence. Other types of involuntary movements of the body and psychiatric illness can also be associated with kifafa. The Wapogoro often relate the condition generally to the occult or more specifically to spirit possession, leading to stigma, namely fear and exclusion of the suffering person. Although views towards epilepsy are slowly changing, fear remains present that contact with bodily excretions of the patient might spread kifafa. Because a close connection exists between NS and epilepsy, the stigma surrounding kifafa is also affecting people with kifafa cha kusinzia.

The Catholic mission runs two shelters near Mahenge for people that cannot provide for themselves due to illness or disability. A large number of people suffering from kifafa are found here, many being excluded by their family. During interviews with community members it was often mentioned that kifafa sufferers from all over the country were brought to these makambi (camps) decades ago, and suspicions were aired that the high prevalence of kifafa in Ulanga district exists because the 'people from the makambi mixed with the local population'.

Several people suffering from kifafa cha kusinzia were visited in the Vigoi division during fieldwork. Especially in more remote villages it was not easy to locate people, because many families live in the hills far from the village centers and main roads. This usually meant a walk through a maze of narrow paths, leading through rice fields and forested hills, with small huts scattered here and there. The difficult access explains why many patients fail to show up for their check-ups at the government hospital. For a while the clinic visited remote areas on a monthly basis to dispense medication, but there is no longer a vehicle available to do this.

The epilepsy clinic did not treat young children with kifafa cha kusinzia at the time of our fieldwork, and in fact had not seen any new cases for a while. All the people we visited at home were above the age of 16 , and most of them above the age of 18 . Only few had cognitive impairments but none of them was attending school; some were sent away out of fear, more often they were advised by teachers to quit because they experienced difficulties with learning. Parents also tend to keep their children at home, fearing that seizures would happen on the way to school on a potentially dangerous place. Like in Kitgum, most of the afflicted people spend their days at home, occasionally doing some small chores. One of them is David (pseudonym), who has been suffering from kifafa cha kusinzia over the past years:

According to interviews during fieldwork, kifafa kuanguka usually raises more fear and is seen as more dangerous. 
In a village one hour away by car from Mahenge, we meet David and his father. David is 21 years old and started experiencing kifafa cha kusinzia about six years ago. He usually gets episodes of head nodding while eating. David's father describes the attacks as 'falling asleep' and 'being unconscious'. Once, he fell down and remained unconscious for hours. After that he started experiencing episodes of head nodding more often, not only during meals. David was still going to school when the problem appeared, but he soon started to forget his books or lose them on the way. He was sent away from school because of fear of transmission. When kifafa cha kusinzia started, the family took him to a traditional healer. According to his father, spirits were involved because David used to be very smart before the nodding started. Unfortunately the treatment did not work and the healer was unable to tell them the cause of the illness. Afterwards, the family consulted their local dispensary and David started taking antiepileptic drugs. Father also mentions a meeting with researchers who suggested that kifafa is caused by an insect. Currently, David experiences around four episodes of head nodding a month. Some people stay away from the family out of fear of contagion and call David names on the street. The family worries about how the illness will progress, since a cure is not known. They state that 'whatever happens in the future is up to God'.

Central in David's story is the search for answers. During the course of the illness, multiple explanatory models circulated. At first, the head nodding episodes were seen as a result of spirit possession, because the attacks started suddenly and David was considered to be healthy before. When a traditional healer failed to bring relief, the family decided to seek help at a medical dispensary. David's father also refers to his interaction with researchers who suggested the involvement of black flies. Currently, people in the area receive yearly treatment for onchocerciasis and David's father expresses hope that therefore there will be less kifafa in the future. But most importantly, the family mentions, it is up to God to decide what will happen.

\section{Lucluc and kifafa cha kusinzia}

The illness narratives in Kitgum and Mahenge show the different and seemingly contradictory discourses and practices that are used when dealing with the syndrome. Biomedical, traditional, and religious discourses and practices are used interchangeably in the search for treatment and explanations. As the case studies illustrate, the socio-political context in which the affliction exists influences explanatory models, case management and illness experience. Irrespective of the different accounts on symptoms, etiology, labelling and treatment, the affected families in both areas mention similar experiences with stigmatization, isolation and a lack of knowledge on cause and cure. Although the content of illness narratives and the management of cases differ in Kitgum en Mahenge, there is constant negotiation about the meaning of terms in both areas.

The illness experience is an important part of the construction of NS and illustrates the intersections of its universalising logic in biomedical terms and the particular context in which the symptoms exist. Unlike the narrow biomedical categorization of nodding syndrome, the concepts of lucluc and kifafa cha kusinzia are able to include narratives on 
important issues that exist in the local context. Here, the body is not understood as a vast and complex machine, but rather as a microcosm of the universe (Scheper-Hughes and Lock 1987, 21). It is necessary to recognize that biomedicine itself is part of an international subculture that imposes certain categories on the world that may not fit equally well everywhere and that never capture the illness experience and concerns of patients (Kirmayer 2001, 27). As Lock and Nguyen $(2010,54)$ argue, the effects of the global dissemination of biomedical knowledge and practice are by no means straightforward. Local accounts, such as given by Deborah and David, should be drawn on in creating health policies, since the promise of and effects of biomedicine are embedded in the social relations and moral landscapes in which they are applied $(2010,5)$. At a time when global discourses on nodding syndrome are predominantly biomedically oriented, we should be careful not to overlook the importance of local conceptualizations in the affected areas.

\section{Conclusion}

The actors, discourses, and practices from the arena of global health were able to cause a shift in meaning through the potential universal validity of their claims: from a type of epilepsy (TZ) or a set of unexplained symptoms (UG) nodding syndrome became recognized as a distinct medical condition. Thereby, transnational intervention spaces came into existence, emphasizing the perceived universal character of NS. The majority of academic literature presents the syndrome as an 'all compassing' phenomenon, whose 'significance and validity is not dependent on the 'props' of a 'culture' or a 'society" (Collier and Ong on global phenomena [2005, 10]). Although biomedicine allows for the possibility that certain afflictions are culture- or context specific, the use of the label and description of NS across different settings illustrates the universality of the constructed medical category of nodding syndrome - in contrast to the variety of concepts circulating 'on the ground'. The aim of this paper has not been to rank the validity of specific claims (or to suggest that non-Western cultures are, by definition, 'traditional' or 'non-scientific' in their explanatory models (Patel 2001, 35)), but to shed light onto the different processes of creating claims, ideas and narratives concerning NS.

The symptoms in Tanzania were known for decades as a specific type of epilepsy and treated as such, but received a new label and level of urgency with the creation of NS as a distinct medical condition. Although attention was paid to the newly constructed diagnosis, a rise in media reports, data on prevalence and a specialized treatment program did not come up. This contrasts with the situation in Uganda, where the symptoms were quickly related to sensitive political issues and received a large amount of national attention. Unlike in Tanzania, differentiation between symptoms of epilepsy and nodding syndrome (plus) takes place in the Ugandan governmental response plan and at the constructed NS treatment centers, resulting in diverse treatment protocols. Following different processes of adaptation, transformation and replacement in society, the syndrome acquired different meanings and management-strategies in Uganda and Tanzania.

Although a new label was created, it did not replace other concepts of the head nodding episodes. Moving away from solely biomedical explanations, this paper shifted from the international definition of nodding syndrome to the concepts of kifafa cha kusinzia and lucluc in the affected districts of Kitgum and Mahenge. Paying attention to the local 
conceptualization of illness is a way to problematize the universality of established Western nosologies. If we accept that there is an authentic illness category, then we expect some actors, beliefs, practices to be 'inside' and that others will be 'outside'. In that case we should only understand the conditions of authenticity in order to determine whether or not it fulfils those conditions (Cook et al. 2009, 66). However, the exploration of different NS-discourses in this paper emphasizes the shifting meaning of the head nodding episodes when dealing with different actors. Even when a universal quality of NS exists, the creation of diagnoses and processes of categorization are still in relation to a common set of meaning, understanding and social structures. Compared to the biomedical definition of NS, the local terminology of lucluc and kifafa cha kusinzia leaves more room for the actual illness experience and context in which it takes place. NS cannot be explained in a complete and unambiguous way because its meaning is continuously being co-produced through interaction, as illustrated by the negotiation of causal factors and inclusion of different types of healers in the illness narratives.

In the end, it is impossible to give an all-encompassing definition of nodding syndrome, lucluc or kifafa cha kusinzia. Although Uganda and Tanzania seem to be affected by a similar set of symptoms, different elements are emphasized which invoke different interpretations, national approaches and processes of conceptualization. Despite the fact that global health actors have powerful voices directing the definition that is used, reconciliation with the specific context of the syndrome has proven to be difficult. Therefore, we must avoid a priori decisions about which kinds of practices and beliefs are part of a universal medical category and be careful not to construct a homogeneous idea of NS. Instead, we suggest to lay to rest holistic assumptions and embrace the indeterminate character of the circulating illness narratives. While acknowledging the fluid meaning of illness, this paper explored the construction of various concepts of 'nodding syndrome' and illustrated how their meaning varies across contexts.

\section{Acknowledgements}

This study is funded by the BOF/Special Research Fund Ghent University, Belgium. Approval for the study was obtained from the Uganda National Council for Science and Technology and the Office of the President and from Muhimbili University of Health and Allied Sciences. We particularly want to thank the people in Uganda and Tanzania who participated in this study.

\section{References}

Bahati L. 2012. Ugonjwa hatari usiotibika, wahofiwa kuingia nchini. Mwananchi, March 17.

Biehl J. and A. Petryna. 2013. Critical Global Health. In When people come first: Critical studies in Global Health, edited by J. Biehl and A. Petryna, 1-20. Princeton: Princeton University Press.

Bukuluki P., I. Ddumba-Nyanzi, J.D. Kisuule, E. Ovuga, L. Lien and D. Kaawa-Mafigiri. 2012. Nodding Syndrome in post conflict northern Uganda: A human security perspective. Global Health Governance VI(1).

Colebunders, R., Hendy, A., Nanyunja, M., Wamalae, J. F. and M. Oijen. 2014. Nodding syndrome - a new hypothesis and new directions for research. International Journal of 
Infectious Diseases 27: 74-77.

Collier, S.J. and A. Ong. 2005. Global Assemblages, Anthropological Problems. In Global Assemblages. Technology, politics and ethics as anthropological problems, edited by

A. Ong and S.J. Collier, 3-21. Malden: Blackwell Publishing.

Conrad P. and K.K. Barker. 2010. The Social Construction of Illness: Key Insights and Policy Implications. Extra Issue, 'What Do We Know? Key Findings from 50 Years of Medical Sociology,' Journal of Health and Social Behavior 51: S67-S79.

Cook, J., J. Laidlaw and J. Mair. 2009. What if There is No Elephant? Towards a Conception of an Un-sited Field. In Multi-Sited Ethnography: Theory, Praxis and Locality in Contemporary Research, edited by M. Falzon, 47-72. Farnham: Ashgate Publishing.

DGHS (Director General of Health Services Uganda), Dr. J.R. Aceng. 2012. Uganda hosts first International Scientific Conference on Nodding Syndrome. Press Release. Kampala 30/07/2012.

Dowell S.F., J.J. Sejvar, L. Riek, K.A.H. Vandemaele, M. Lamunu, A.C. Kuesel, E. Schmutzhard, et al. 2013. Nodding Syndrome. Emerging Infectious Diseases 19 (9): 1374-1384.

Fassin D. 2011. Coming Back to Life: An Anthropological Reassessment of Biopolitics and Governmentality. In Governmentality: Current Issues and Future Challenges, edited by U. Bröckling, S. Krasmann and T. Lemke, 165-184. New York: Routledge.

Fuller S. 2015. Knowledge. The Philosophical Quest in History. Oxon: Routledge.

Hanna B. and A. Kleinman. 2013. Unpacking Global Health: Theory and Critique. In Reimagining Global Health: An Introduction, edited by P. Farmer, J. Yong Kim, A. Kleinman and M. Basilico, 15-32. Berkeley: University of California Press.

Idro R., K.A. Musubire, B. Byamah Mutamba, H. Namusoke, J. Muron, C. Abbo, R. Oriyabuzu et al. 2013. Proposed guidelines for the management of nodding syndrome. African Health Sciences 13 (2): 219-323.

Iyengar P.J., J. Wamala, J. Ratto, C. Blanton, M. Malimbo, L. Lukwago, S. Becknell et al. 2014. Prevalence of Nodding Syndrome - Uganda 2012-2013. Morbidity and Mortality Weekly Report (CDC) 63 (28): 603-606.

Jilek W.G. and L.M. Jilek-Aall. 1970. The problem of epilepsy in rural Africa: 'Kifafa' in a Tanzanian tribe. Paper presented at the Colloque de l'Epidemiologue de l'Epilepsie en Afrique, Marseille, 3 September 1968. Transcultural Psychiatry 7: 43-48.

Kirmayer J.L. 2001. Cultural Variations in the Clinical Presentation of Depression and Anxiety: Implications for Diagnosis and Treatment. Journal of Clinical Psychiatry 62 (13): 22-28.

Korevaar D.A. and B.J. Visser. 2012. Reviewing the evidence on nodding syndrome, a mysterious tropical disorder. International Journal of Infectious Diseases 17 (3): 149452.

Langwick S.A., H. Dilger and A. Kane. 2012. Introduction: Transnational Medicine, Mobile Experts. In Medicine, Mobility, and Power in Global Africa: Transnational Health and Healing, edited by H. Dilger, A. Kane and S. Langwick, 1-27. Bloomington: Indiana University Press.

Lewis-Fernández, R. 1996. Cultural formulation of psychiatric diagnosis. Culture, Medicine and Psychiatry 20: 133-144. 
Lock, M. and Nguyen V. 2010. An anthropology of biomedicine. Malden: Wiley-Blackwell. Marcus G.E. 1995. Ethnography in/of the World System: The Emergence of Multi-Sited Ethnography. Annual review of anthropology 24 (1): 95-117.

Ministry of Health Uganda. 2012a 'Nodding Syndrome Health Workers' Training Manual. Revised Edition: August 2012.

Ministry of Health Uganda. 2012b. Integrated Response Plan for the Control of the Nodding Disease Syndrome in Acholi Sub-region, Uganda. January 2012 to December 2014. The Government of Uganda. January 2012.

Mitchell K.B., J. Kornfeld, J. Adiama, A. Mugenyi, E. Schmutzhard, E. Ovuga, J. Kamstra et al. 2013. Nodding Syndrome in Northern Uganda: Overview and Community Perspectives. Epilepsy \& Behaviour 26 (1): 22-24.

Mugerwa Y. and A. Ayebazibwe. 2012. Uganda state house asks for cash to tackle disease. The Citizen, February 18.

Musisi S., D. Akena, E. Nakimuli-Mpungu, C. Abbo and J. Okello. 2013. Neuropsychiatric perspectives on nodding syndrome in northern Uganda: a case series study and a review of the literature. African Health Sciences 13 (2): 205-218.

Okony Tumanu Tim. 2014a. Song: 'Two oneko latina'. Accessed October 2015. http://www.ugandanrecordings.com/okony-tumangu-tim-two-oneko-latina/

Okony Tumanu Tim. 2014b. Song: 'Aculube ocwalo kwena'. Accessed October 2015. http://www.ugandanrecordings.com/okony-tumangu-tim-aculube-ocwalokwena-english-subtitles/

Okony Tumanu Tim. 2014c. Song: 'Two lucluc'. Accessed October 2015. http://www.ugandanrecordings.com/okony-tumangu-tim-two-lucluc-english-subtitles/

Patel, V. 2001. Cultural factors and international epidemiology. Depression and public health. British Medical Bulletin 57 (1): 33-45.

Scheper-Hughes, N. and M.M. Lock. 1987. The Mindful Body: A Prolegomenon to Future Work in Medical Anthropology. Medical Anthropology Quarterly 1 (1): 6-41.

Sluka J.A. and A.C.G.M. Robben. 2012. Fieldwork in Cultural Anthropology: An Introduction. In Ethnographic Fieldwork: An Anthropological Reader. $2^{\text {nd }}$ edition, edited by A.C.G.M. Robben and J. A. Sluka, 1-47. Hoboken: John Wiley \& Sons Inc.

Spencer P.S., K. Vandemaele, M. Richer, V.S. Palmer, S. Chungong, M. Anker, Y. Ayana et al. 2013. Nodding Syndrome in Mundri county, South Sudan: environmental, nutritional and infectious factors. African Health Sciences 13 (2): 183-204.

Tumwine J.K., K. Vandemaele, S. Chungong, M. Richer, M. Anker, Y. Ayana, M.L. Opoka et al. 2012. Clinical and epidemiological characteristics of nodding syndrome in Mundri County, southern Sudan. African Health Sciences 12 (3): 242-248.

Van Bemmel K., I. Derluyn and K. Stroeken. 2014. Nodding Syndrome or Disease? On the Conceptualization of an Illness-in-the-making. Ethnicity \& Health 19 (1): 100-118.

Van Bemmel K. 2016a. The rise and fall of nodding syndrome in public discourse: an analysis of newspaper coverage in Uganda. Critique of Anthropology 36 (2): 168-196.

Van Bemmel K. 2016b. The Quest for treatment: The violated body of nodding syndrome in northern Uganda. Journal of Peace and Security Studies 2(2): 63-78.

Van Bemmel, K. and E.D. Aalten (Producers). 2016. Nodding Syndrome; an illness in the 
making (Documentary). The Netherlands: Scandal Studios.

Winkler A.S., K. Friedrich, R. König, M. Meindl, R. Helbok, I. Unterberger, T. Gotwald et al. 2008. The head nodding syndrome - clinical classification and possible causes. Epilepsia 49 (12): 2008-2015.

Winkler, A.S., B. Wallner, K. Friedrich, B. Pfausler, I. Unterberger, W. Matuja, L. Jilek-Aall et al. 2014. A longitudinal study on nodding syndrome: A new African epilepsy disorder. Epilepsia. 55 (1): 86-93.

WHO (World Health Organization). 2005. Health and Mortality Survey among Internationally Displaced Persons in Gulu, Kitgum and Pader Districts, Northern Uganda.

WHO (World Health Organization). 2012. International Scientific Meeting on Nodding Syndrome. Meeting Report. UKaid, CDC, The Republic of Uganda, WHO, Kampala 30 July-1 August. 